\title{
6-Hydroxythiobinupharidine Inhibits Migration of LM8 Osteosarcoma Cells by Decreasing Expression of LIM Domain Kinase 1
}

\author{
MASATO YOSHIZAWA ${ }^{1}$, SEIKOU NAKAMURA ${ }^{2}$, YUKI SUGIYAMA ${ }^{1}$, SHIORI TAMAI $^{1}$, YUKIKO ISHIDA ${ }^{1}$, \\ MARI SUEYOSHI ${ }^{1}$, YUKI TODA ${ }^{1}$, SHIGEKUNI HOSOGI ${ }^{1}$, YOSHITAKA YANO $^{3}$ and EISHI ASHIHARA ${ }^{1}$ \\ ${ }^{1}$ Department of Clinical and Translational Physiology, Kyoto Pharmaceutical University, Kyoto, Japan; \\ ${ }^{2}$ Department of Pharmacognosy, Kyoto Pharmaceutical University, Kyoto, Japan; \\ ${ }^{3}$ Education and Research Center for Clinical Pharmacy, Kyoto Pharmaceutical University, Kyoto, Japan
}

\begin{abstract}
Background/Aim: Osteosarcoma is the most malignant type of bone tumor. Patients with osteosarcoma metastases have a poorer prognosis than those without metastases. Thus, the prognosis of osteosarcoma patients with metastases must be improved. Materials and Methods: The present study investigated the inhibitory effects of 6hydroxythiobinupharidine isolated from Nuphar pumilum on migration of LM8 murine osteosarcoma cells by a migration assay and also examined the expression of proteins related to actin dynamics by western blot. The present study also developed an automatic cell counting system using machine learning to count migrated cells by Fiji and Trainable Weka Segmentation. Results: 6-Hydroxythiobinupharidine inhibited migration of LM8 osteosarcoma cells in a dose-dependent manner, and decreased protein expression of Lin11, Isl-1, and Mec-3 domain kinase 1 (LIMK1) and the levels of phosphorylated Cofilin. Conclusion: 6-Hydroxythiobinupharidine suppressed migration of LM8 osteosarcoma cells by decreasing expression of LIMK1. 6-Hydroxythiobinupharidine could be potentially used as an anti-metastatic compound.
\end{abstract}

Osteosarcoma is the most malignant type of bone tumors and preferentially develops in young and elderly patients (1). Patients with localized osteosarcoma are expected to be cured by surgery and chemotherapy; however, it is difficult to cure osteosarcoma patients with distant metastases (2). In particular, osteosarcoma patients with lung metastases have a poor prognosis and their 5-year survival rate is lower than

Correspondence to: Eishi Ashihara, MD, Ph.D., 5 Nakauchi-cho, Misasagi, Yamashina, Kyoto, 607-8414, Japan. Tel: +81 755954705, Fax: +81 755954796, e-mail: ash@mb.kyoto-phu.ac.jp

Key Words: Osteosarcoma, 6-hydroxythiobinupharidine, metastasis, LIMK, Cofilin, machine learning. that of osteosarcoma patients without metastases $(3,4)$. Therefore, effective agents to inhibit osteosarcoma metastases must be identified.

The nupharidine compound 6-hydroxythiobinupharidine is the main constituent of the rhizome of Nuphar pumilum. Recent studies have shown that nupharidine compounds have anti-cancer and anti-metastatic effects $(5,6)$. This study aimed to investigate the inhibitory effects of 6-hydroxythiobinupharidine on the migration of LM8 osteosarcoma cells and to elucidate the underlying mechanism.

\section{Materials and Methods}

Cell culture and preparation of 6-hydroxythiobinupharidine. The LM8 murine osteosarcoma cell line was established from the Dunn osteosarcoma cell line and has a high lung metastatic potential (7). These cells were cultured in high-glucose Dulbecco's modified Eagle's medium (DMEM; Nacalai Tesque, Kyoto, Japan) containing $10 \%$ heat-inactivated fetal bovine serum (Sigma-Aldrich, St. Louis, MO, USA) and $1 \%$ penicillin-streptomycin (Thermo Fisher Scientific, Tokyo, Japan) and maintained in a fully humidified incubator at $37^{\circ} \mathrm{C}$ with $5 \% \mathrm{CO}_{2} .6-\mathrm{Hydroxythiobinupharidine} \mathrm{was}$ isolated from the rhizome of $N$. pumilum by the Department of Pharmacognosy at Kyoto Pharmaceutical University. It was dissolved in dimethyl sulfoxide (FUJIFILM Wako Pure Chemical Corporation, Osaka, Japan) to a concentration of $100 \mathrm{mM}$, and stored at $-30^{\circ} \mathrm{C}$.

WST-8 assay. The cytotoxic effect of 6-hydroxythiobinupharidine on LM8 cells was investigated by the WST-8 assay using a Cell Counting Kit-8 (DOJINDO, Kumamoto, Japan) as previously reported (8). In brief, $3 \times 10^{3} \mathrm{LM} 8$ cells were seeded into each well of a flat-bottomed 96-well plate (Thermo Fisher Scientific) in $100 \mu \mathrm{l}$ of medium and treated with various concentrations $(0.1,1,3$, and $10 \mu \mathrm{M})$ of 6 hydroxythiobinupharidine or remained untreated $(0 \mu \mathrm{M})$ for $72 \mathrm{~h}$.

Fiji and Trainable Weka Segmentation (TWS; ver.3.2.33). Fiji (9) is an alternative to ImageJ with many plugins to analyze scientific images (10). Fiji (Windows platform) was downloaded and installed 
from (9). TWS is a Fiji plugin that combines a collection of machine learning algorithms with a set of selected image features to generate pixel-based segmentations (11).

Development of an automatic cell counting system (ACCS) with machine learning. Migrated cells needed to be counted in the migration assay. However, there is no system to automatically count such cells. Therefore, an ACCS was developed using Fiji and a machine learning method (TWS). To evaluate the practicality of the ACCS, 15 randomly chosen images from the migration assay were used for learning and 36 and 348 images were used for testing. TWS was performed using 15 randomly chosen images as learning data from among many images of the migration assay. Based on this, an ACCS was developed. The machine learning system recognized the number of nuclei as the number of cells. Cells in 36 images of the migration assay were manually counted by five investigators and automatically counted by the ACCS. The 36 average values generated by the five investigators and the 36 values generated by the ACCS were determined. Thereafter, cells in 348 images of the migration assay were manually counted by one investigator and automatically counted by the ACCS. The 348 values generated by the one investigator and the 348 values generated by the ACCS were determined. The values obtained by manual counting and those using the ACCS were divided by the average number of cells counted upon treatment with $0 \mu \mathrm{M} 6$ hydroxythiobinupharidine and converted into percentages using Microsoft Excel for Windows 2019. The correlation and slope, correlation coefficient (r), 95\% confidence interval (CI) of the slope, and $r$ for each percentage value when comparing manual counting and the ACCS were determined using the 36 and 348 images.

Cell migration assay. LM8 cells $\left(3.0 \times 10^{4}\right.$ cells $/ \mathrm{ml}$ in serum-free DMEM) were seeded into cell culture inserts containing pores with a diameter of $8 \mu \mathrm{m}$ (BD Biosciences, San Jose, NJ, USA). The inserts were placed in 24-well companion plates (BD Biosciences) with DMEM containing $1 \%$ fetal bovine serum as a chemoattractant. 6-Hydroxythiobinupharidine was added to the insert at various concentrations $(0.1$ and $1 \mu \mathrm{M})$ or not added $(0 \mu \mathrm{M})$. After $24 \mathrm{~h}$, non-migrated cells in the upper chamber were removed with a cotton swab. Migrated cells in the bottom chamber were fixed with $100 \%$ methanol for 2 min and stained with Giemsa stain solution (Wako, Osaka, Japan) for $15 \mathrm{~min}$. Images of migrated cells in three randomly chosen fields (40× magnification) per insert were acquired under a BX50 light microscope (Olympus, Tokyo, Japan) equipped with a DP72 digital camera (Olympus). Migrated cells in these images were counted using the ACCS.

Western blot analysis. LM8 cells were seeded into 6-cm dishes (Thermo Fisher Scientific) at a density of $3.0 \times 10^{4}$ cells $/ \mathrm{ml}$. After treatment with or without 6-hydroxythiobinupharidine for $2 \mathrm{~h}$, LM8 cells were collected as described previously (8). Samples (20 $\mu \mathrm{g}$ of protein) were blotted with primary antibodies against the following proteins: cell division cyclin 42 (Cdc42; Cell Signaling Technology, Beverly, MA, USA), Ras-related C3 botulinus toxin substrate 1 (Rac1; BD Pharmingen, San Jose, CA, USA), Rho family small GTPbinding protein A (RhoA; Santa Cruz Biotechnology, Dallas, TX, USA), Rho-associated protein kinase 1 (ROCK1; BD Pharmingen), LIMK1 (Cell Signaling Technology), Cofilin and phospho-Cofilin (Ser3) (Cell Signaling Technology), and glyceraldehyde-3-phosphate dehydrogenase (GAPDH; Cell Signaling Technology). Horseradish
Table I. Correlation analysis of manual counting and the automatic cell counting system using 36 images.

\begin{tabular}{lc}
\hline Slope & 0.996 \\
(95\% confidence interval) & $(0.976-1.015)$ \\
& \\
Correlation coefficient & 0.998 \\
$(95 \%$ confidence interval) & $(0.997-0.999)$ \\
$p$-Value (two-tailed) & $<0.0001$ \\
\hline
\end{tabular}

Table II. Correlation analysis of manual counting and the automatic cell counting system using 348 images.

\begin{tabular}{lc}
\hline Slope & 0.950 \\
(95\% confidence interval) & $(0.929-0.972)$ \\
& \\
Correlation coefficient & 0.978 \\
$(95 \%$ confidence interval) & $(0.973-0.982)$ \\
$p$-Value (two-tailed) & $<0.0001$ \\
\hline
\end{tabular}

peroxidase-coupled anti-rabbit and anti-mouse IgGs were used as secondary antibodies. Bands corresponding to immunoreactive proteins were visualized with Amersham ECL or an ECL Prime Western Blotting Detection Kit (GE Healthcare, Little Chalfont, UK).

Statistical analysis. Data are presented as means+standard errors of the means (SEM) of three independent experiments. All analyses were conducted using GraphPad Prism 5 (GraphPad Software Inc., San Diego, CA, USA). Three or more groups were statistically compared using a one-way analysis of variance (ANOVA) and multiple comparisons of means were performed with Bonferroni's correction. $p<0.05$ was considered significant. The significance of the $r$ values when comparing manual counting and the ACCS using 36 and 348 images was determined by calculating the Pearson correlation using GraphPad Prism 5. The $p$-value of the $r$ value indicated whether it significantly differed from zero. $p<0.05$ was considered significant.

\section{Results}

Cell counting using the ACCS correlates with manual counting. We analyzed the correlation and slope, r, 95\% CI of the slope, and $r$ for each percentage value when comparing manual counting and the ACCS using 36 images (Figure 1A and Table I) and 348 images (Figure 1B and Table II). There was a high correlation when comparing each percentage value between manual counting and the ACCS using 36 images $(\mathrm{r}=0.998 ; p<0.0001)$ and 348 images $(\mathrm{r}=0.978 ; p<0.0001)$. Thus, the ACCS generated similar results as manual counting and was therefore used for subsequent measurements. 

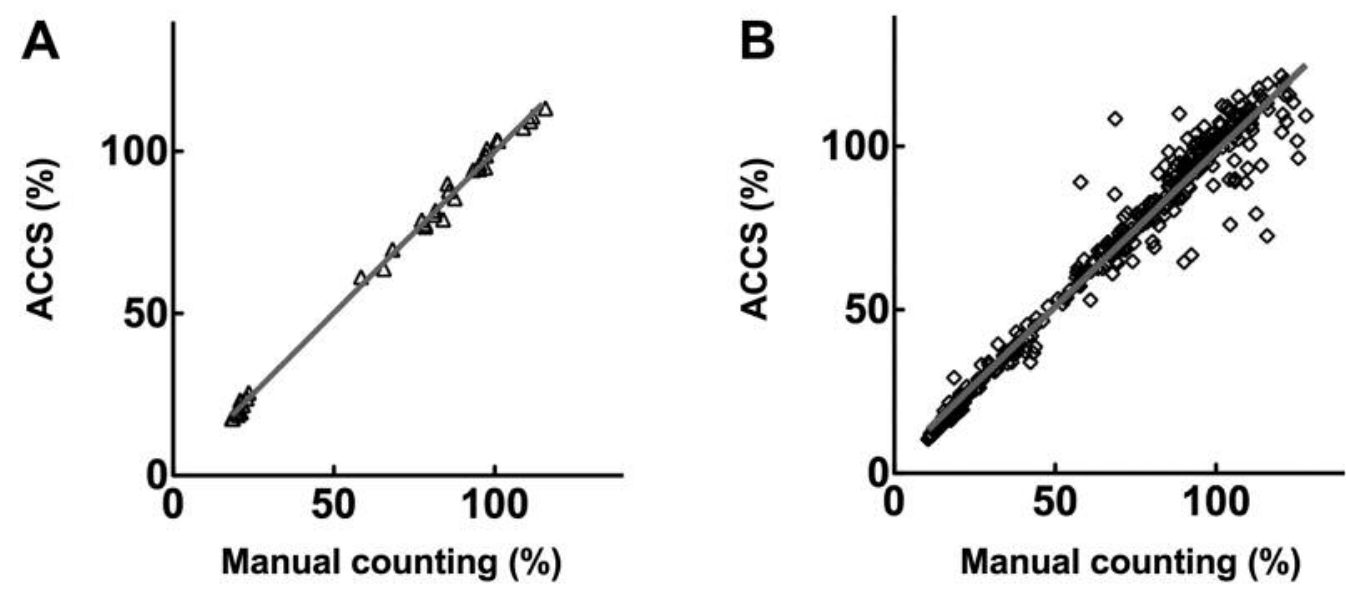

Figure 1. Comparison of the ACCS and manual counting. (A) Correlation analysis of manual counting and the ACCS using 36 images (r=0.998, $p<0.0001)$. The gray line is the slope (slope=0.996). (B) Correlation analysis of manual counting and the ACCS using 348 images $(r=0.978$, $p<0.0001$ ). The gray line is the slope (slope=0.950). The significance of the $r$ values between manual counting and the ACCS using 36 and 348 images was determined by calculating the Pearson correlation using GraphPad Prism 5. The p-value of the $r$ value indicated whether it significantly differed from zero. $p<0.05$ was considered significant.

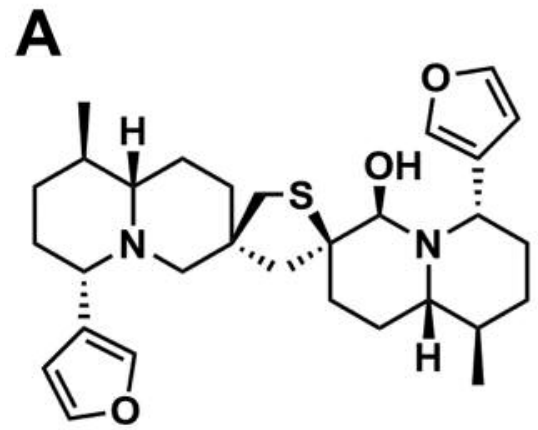

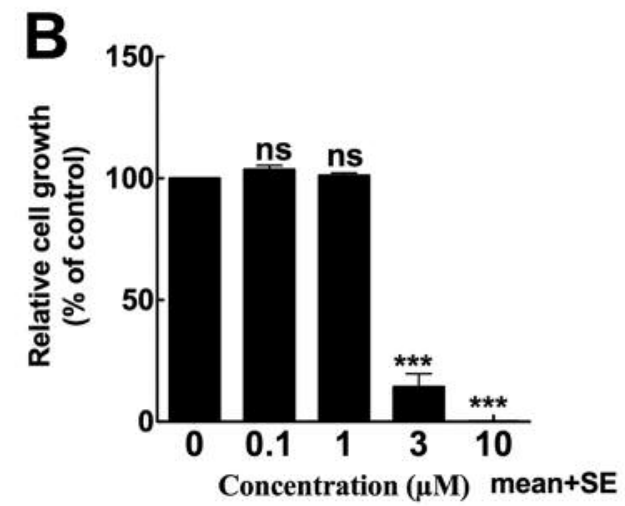

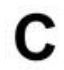

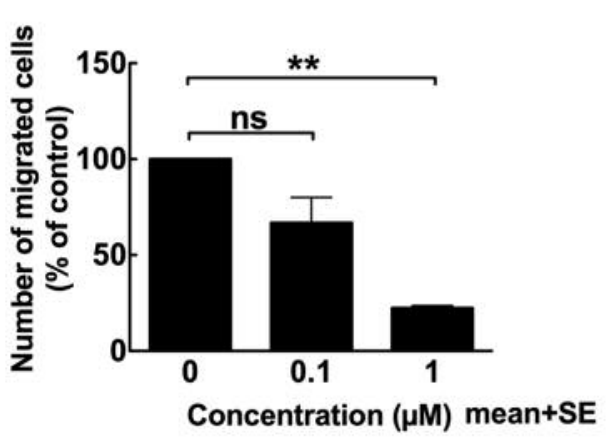

D

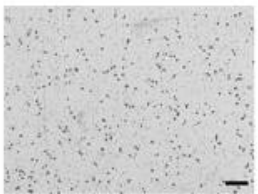

0

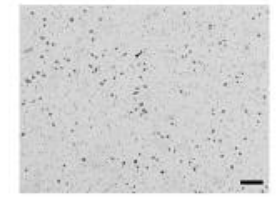

0.1

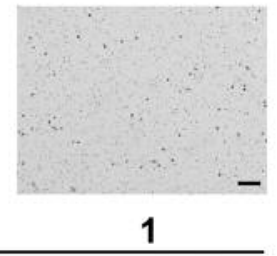

6-Hydroxythiobinupharidine $(\mu \mathrm{M})$

Figure 2. Results of the WST-8 and migration assays. (A) Structure of 6-hydroxythiobinupharidine. (B) Cytotoxic effects of 6-hydroxythiobinupharidine on LM8 cells in the WST-8 assay. LM8 cells were treated with various concentrations $(0.1,1,3$, and $10 \mu M)$ of 6-hydroxythiobinupharidine or not treated (0 $\mu M)$ for $72 \mathrm{~h}$. Data represent the mean+SEM of three independent experiments. Means+SEM were analyzed using a one-way ANOVA and multiple comparisons of means were performed with Bonferroni's correction. $p<0.05$ was considered significant. ns: Not significant; ***p<0.001. (C) Inhibitory effect of 6-hydroxythiobinupharidine on migration of LM8 cells as shown in the migration assay. LM8 cells were treated with various concentrations (0.1 and $1 \mu M)$ of 6-hydroxythiobinupharidine or not treated $(0 \mu M)$ for 24 h. Data represent the mean + SEM of three independent experiments. Means + SEM were analyzed using a one-way ANOVA and multiple comparisons of means were performed with Bonferroni's correction. p<0.05 was considered significant. ns: Not significant; **p<0.01. (D) Images of migrated cells after treatment with or without 6-hydroxythiobinupharidine for 24 h. Scale bars: 200 um. 


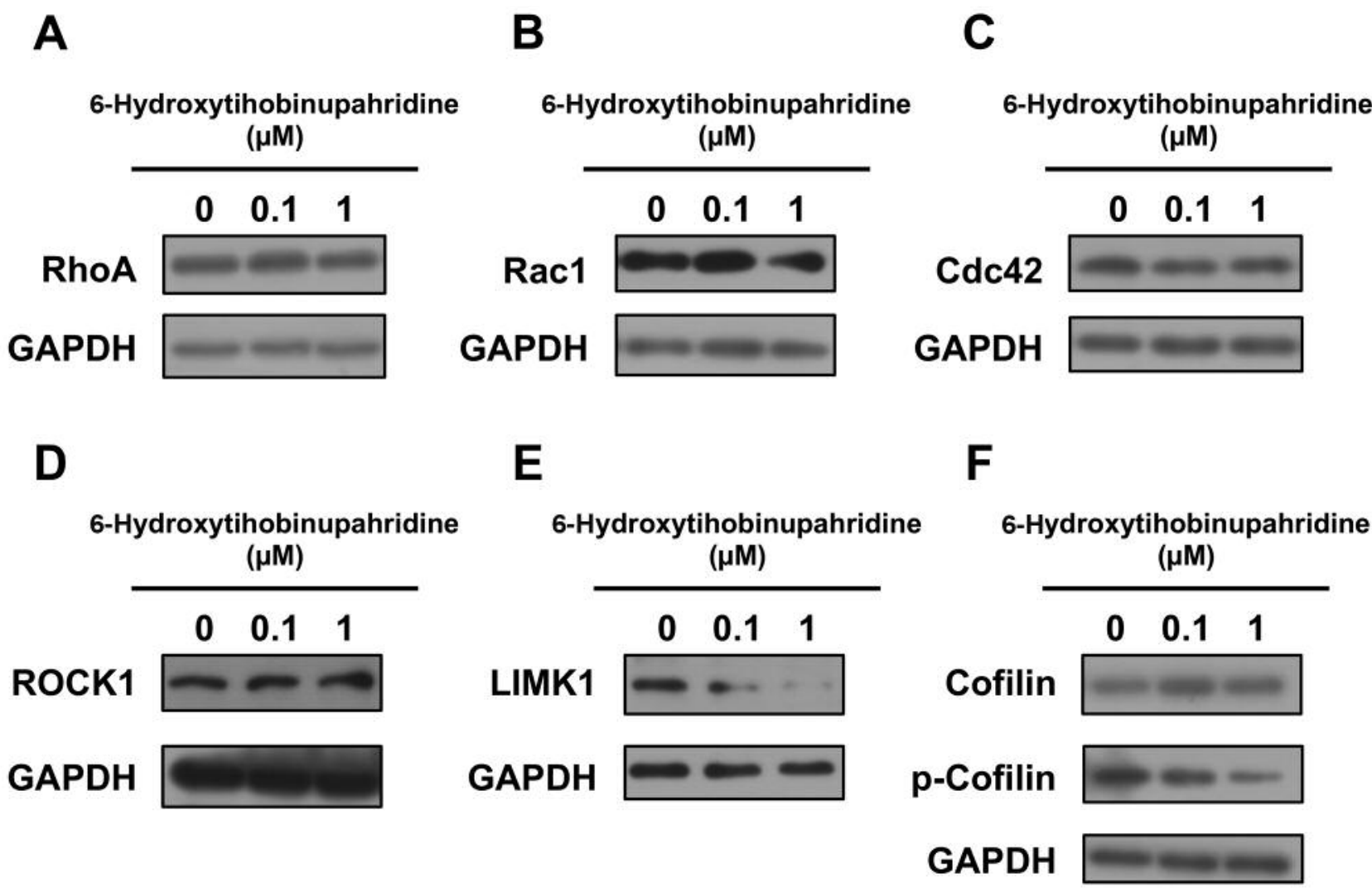

Figure 3. Effects of 6-hydroxythiobinupharidine on protein expression in LM8 cells. LM8 cells were treated with various concentrations (0.1 and 1 $\mu M)$ of 6-hydroxythiobinupharidine or not treated $(0 \mu M)$ for $2 \mathrm{~h}$. Thereafter, the expression levels of (A) RhoA, (B) Rac1, (C) Cdc42, (D) ROCK1, (E) LIMK1, $(F)$ phosphorylated Cofilin and Cofilin were assessed by western blotting. GAPDH was used as a loading control.

Determination of 6-hydroxythiobinupharidine concentrations that do not affect growth of LM8 cells. The WST-8 assay was conducted to investigate whether 6-hydroxythiobinupharidine (Figure 2A) affected growth of LM8 cells. Cell growth was inhibited by treatment with 6-hydroxythiobinupharidine at concentrations of $3 \mu \mathrm{M}$ or higher, but not at concentrations of $1 \mu \mathrm{M}$ or lower (Figure 2B). Therefore, we treated cells with 0.1 and $1 \mu \mathrm{M}$ 6-hydroxythiobinupharidine in the migration assay to investigate the direct effects of this compound on cell migration.

6-Hydroxythiobinupharidine inhibits migration of LM8 cells. Migration assays were conducted to investigate the inhibitory effects of 6-hydroxythiobinupharidine on migration of LM8 cells. Treatment with 6-hydroxythiobinupharidine at concentrations that did not affect cell growth inhibited migration of LM8 cells in a dose-dependent manner (Figure 2C, D).

6-Hydroxythiobinupharidine decreases the expression levels of LIMK1 and phosphorylated Cofilin. Cell migration involves polymerization and depolymerization of actin $(12,13)$, which are controlled by Cdc42, Rac, Rho, LIMK, and Cofilin (1416). Further, by decreasing expression of $\mathrm{Cdc} 42$, the motility of LM8 cells was decreased (17). We hypothesized that the inhibitory effect of 6-hydroxythio-binupharidine on the migration of LM8 cells involved actin polymerization, actin depolymerization, and actin cytoskeleton formation. Therefore, western blotting was conducted to investigate whether 6-hydroxythiobinupharidine affected the expression levels of RhoA, Rac1, Cdc42, ROCK1, LIMK1, Cofilin, and phosphorylated Cofilin (Ser3). 6-Hydroxythio-binupharidine decreased the expression levels of LIMK1 and phosphorylated Cofilin, but did not change those of RhoA, Rac1, Cdc42, ROCK1, or Cofilin (Figure 3A-F).

\section{Discussion}

In this study, we developed an ACCS using machine learning as a cell counting method that was independent of investigators to obtain consistent results in the migration assay. Cell counting is important in migration assays. However, this process is 


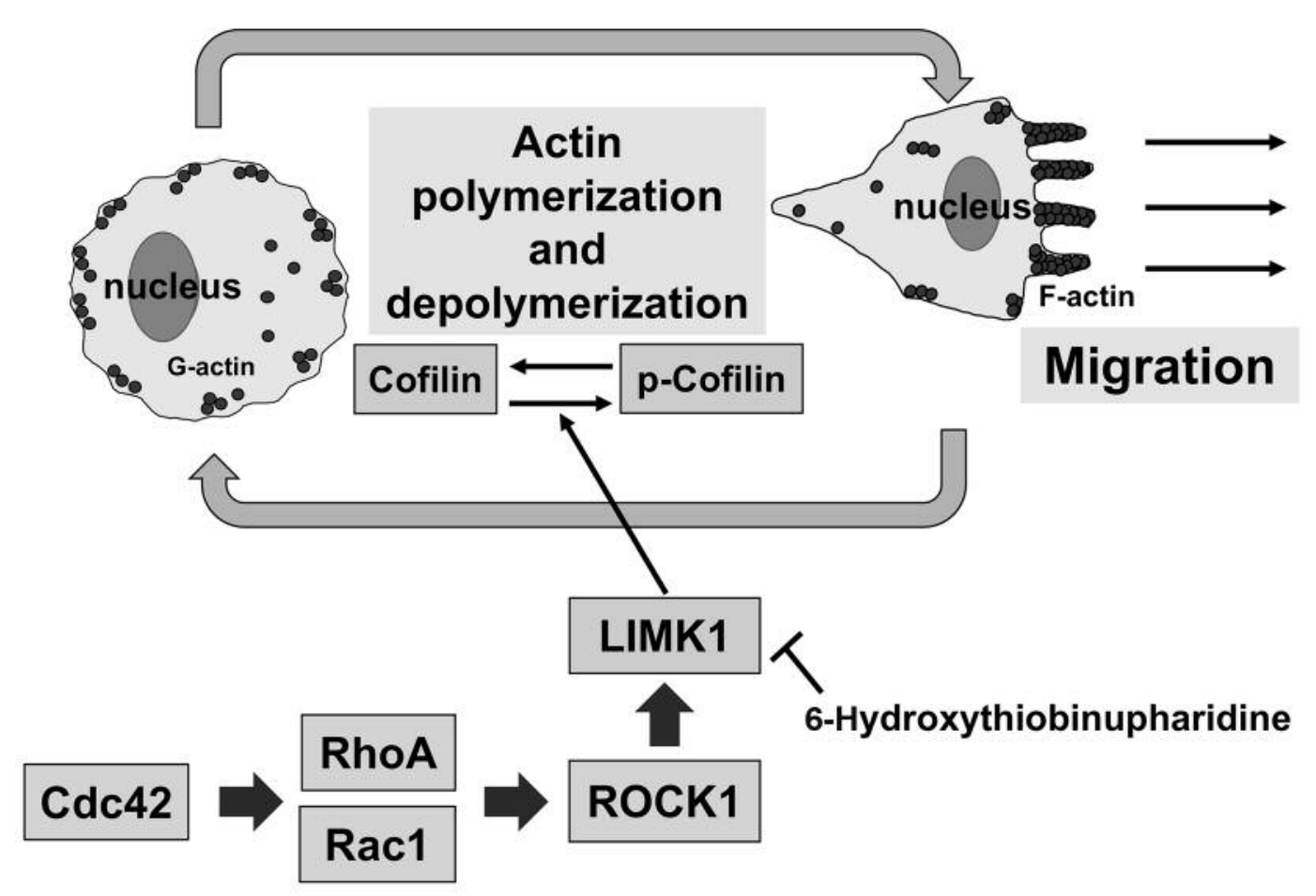

Figure 4. A model of the mechanism by which 6-hydroxythiobinupharidine inhibits cell migration. 6-Hydroxythiobinupharidine decreases expression of LIMK1 and thus the levels of phosphorylated Cofilin. As a result, actin regulation is disrupted and cell migration is inhibited.

easily influenced by the experience and technique of investigators, and it is difficult to ensure the accuracy and reproducibility of results between different investigators. Therefore, we developed an ACCS that recognized nuclei using machine learning to count the number of nuclei as the number of cells. Evaluation of the correlation of each percentage value between manual counting and the ACCS demonstrated the practicality of this system. We believe that the ACCS provides consistent cell counts in migration assays. The source code and learning data of the ACCS are available at (18).

We next investigated whether 6-hydroxythiobinupharidine inhibits migration of LM8 osteosarcoma cells using transwell chambers and explored the underlying mechanism by performing western blotting. Nupharidine compounds from rhizomes of $N$. pumilum have several biological activities including immunosuppressive, anti-metastatic, and rapid apoptosis-inducing effects (19). 6-Hydroxythiobinupharidine is a nupharidine compound. Nuphar lutea leaf extracts elicit antimetastatic effects on the human alveolar adenocarcinoma A549 cell line and the human breast cancer MCF-7 cell line by inhibiting nuclear factor- $\mathrm{kB}(6)$. Reorganization of the actin cytoskeleton plays important roles in cell migration and involves many molecules (20). LIMK1 and Cofilin are important molecules that regulate actin polymerization and depolymerization (21). The serine/threonine kinase LIMK1 participates in the regulation of the actin cytoskeleton architecture and actin dynamics by phosphorylating Cofilin at Ser3 (22). Cofilin is involved in polymerization and depolymerization of actin (23). Activation of Cofilin via inhibition of LIMK by cucurbitacin E suppressed migration of intestinal epithelial cells in vitro by disrupting actin dynamics (24). MicroRNAs targeting LIMK1 or components of the LIMK1/CFL1 pathway suppressed the motility of breast cancer cells $(25,26)$. Down-regulation of Cofilin by alendronate in PC3 prostate cancer cells disrupted the actin cytoskeleton and inhibited migration (27). The current study showed that 6hydroxythiobinupharidine inhibited migration of LM8 osteosarcoma cells and decreased the expression levels of LIMK1 and phosphorylated Cofilin, which is a novel discovery.

In conclusion, our results suggest the nupharidine compound 6-hydroxythiobinupharidine inhibits migration of LM8 osteosarcoma cells by decreasing the expression levels of LIMK1 and phosphorylated Cofilin. We consider that 6hydroxythiobinupharidine decreases expression of LIMK1 and thereby the levels of phosphorylated Cofilin. In this way, we believe that 6-hydroxythiobinupharidine disrupts actin regulation and inhibits cell migration (Figure 4). Thus, 6hydroxythiobinupharidine can effectively inhibit the formation of metastases by inhibiting cell migration, which is the first stage of metastasis. 


\section{Conflicts of Interest}

All Authors have no conflict of interest to disclose regarding this study.

\section{Author's Contributions}

M.Y. performed experiments and analyzed data; M.Y., Y.S., S.T., Y.I., and M.S. performed manual counting and developed the ACCS; S.N. provided 6-hydroxythiobinupharidine; S.N, Y.T., S.H., Y.Y., and E.A. provided intellectual guidance; M.Y. and E.A. wrote the article.

\section{Acknowledgements}

This work was partly supported by Grant-in-Aids for Scientific Research from the Ministry of Education, Culture, Sports, Science, and Technology of Japan (MEXT, 26461436 and 19K08826 to EA) and the MEXT-Supported Program for the Strategic Research Foundation at Private Universities, 2015-2019 (S1511024L to EA).

\section{References}

1 Mirabello L, Troisi RJ and Savage SA: Osteosarcoma incidence and survival rates from 1973 to 2004: Data from the Surveillance, Epidemiology, and End Results Program. Cancer 115(7): 15311543, 2009. PMID: 19197972. DOI: 10.1002/cncr.24121

2 Gill J, Ahluwalia MK, Geller D and Gorlick R: New targets and approaches in osteosarcoma. Pharmacol Ther 137(1): 89-99, 2013. PMID: 22983152. DOI: 10.1016/j.pharmthera.2012.09.003

3 Huang X, Zhao J, Bai J, Shen H, Zhang B, Deng L, Sun C, Liu $\mathrm{Y}$, Zhang $\mathrm{J}$ and Zheng J: Risk and clinicopathological features of osteosarcoma metastasis to the lung: A population-based study. J Bone Oncol 16: 100230, 2019. PMID: 30923668. DOI: 10.1016/j.jbo.2019.100230

4 Kaste SC, Pratt CB, Cain AM, Jones-Wallace DJ and Rao BN: Metastases detected at the time of diagnosis of primary pediatric extremity osteosarcoma at diagnosis: imaging features. Cancer 86(8): 1602-1608, 1999. PMID: 10526292. DOI: 10.1002/ (sici)1097-0142(19991015)86:8<1602::aid-cncr31>3.0.co;2-r

5 Ozer J, Eisner N, Ostrozhenkova E, Bacher A, Eisenreich W, Benharroch D, Golan-Goldhirsh A and Gopas J: Nuphar lutea thioalkaloids inhibit the nuclear factor карраB pathway, potentiate apoptosis and are synergistic with cisplatin and etoposide. Cancer Biol Ther 8(19): 1860-1868, 2014. PMID: 19713755. DOI: $10.4161 / \mathrm{cbt} .8 .19 .9567$

6 Ozer J, Fishman D, Eilam B, Golan-Goldhirsh A and Gopas J: Anti-metastatic effect of semi-purified Nuphar lutea leaf extracts. J Cancer 8(8): 1433-1440, 2017. PMID: 28638458. DOI: $10.7150 /$ jca. 18435

7 Asai T, Ueda T, Itoh K, Yoshioka K, Aoki Y, Mori S and Yoshikawa $\mathrm{H}$ : Establishment and characterization of a murine osteosarcoma cell line (LM8) with high metastatic potential to the lung. Int J Cancer 76(3): 418-422, 1998. PMID: 9579581. DOI: 10.1002/(sici)10970215(19980504)76:3<418::aid-ijc21>3.0.co;2-5

8 Fukuda H, Nakamura S, Chisaki Y, Takada T, Toda Y, Murata H, Itoh K, Yano Y, Takata K and Ashihara E: Daphnetin inhibits invasion and migration of LM8 murine osteosarcoma cells by decreasing RhoA and Cdc42 expression. Biochem Biophys Res
Commun 471(1): 63-67, 2016. PMID: 26845352. DOI: 10.1016/j.bbrc.2016.01.179

9 Fiji: ImageJ, with "Batteries Included". Available at https://fiji.sc (Last accessed on 12 November 2019)

10 Schindelin J, Arganda-Carreras I, Frise E, Kaynig V, Longair M, Pietzsch T, Preibisch S, Rueden C, Saalfeld S, Schmid B, Tinevez JY, White DJ, Hartenstein V, Eliceiri K, Tomancak P and Cardona A: Fiji: an open-source platform for biologicalimage analysis. Nat Methods 9(7): 676-682, 2012. PMID: 22743772. DOI: $10.1038 /$ nmeth.2019

11 Arganda-Carreras I, Kaynig V, Rueden C, Eliceiri KW, Schindelin J, Cardona A and Sebastian Seung H: Trainable Weka Segmentation: a machine learning tool for microscopy pixel classification. Bioinformatics 33(15): 2424-2426, 2017. PMID: 28369169. DOI: 10.1093/bioinformatics/btx 180

12 Carlier MF, Le Clainche C, Wiesner S and Pantaloni D: Actinbased motility: from molecules to movement. Bioessays 25(4): 336-345, 2003. PMID: 12655641. DOI: 10.1002/bies.10257

13 Pollard TD and Borisy GG: Cellular motility driven by assembly and disassembly of actin filaments. Cell 112(4): 453-465, 2003. PMID: 12600310. DOI: 10.1016/s0092-8674(03)00120-x

14 Carlier MF, Ressad F and Pantaloni D: Control of actin dynamics in cell motility. Role of ADF/cofilin. J Biol Chem 274(48): 33827-33830, 1999. PMID: 10567336. DOI: 10.1074/ jbc.274.48.33827

15 Hall A: Rho GTPases and the actin cytoskeleton. Science 279(5350): 509-514, 1998. PMID: 9438836. DOI: 10.1126/ science.279.5350.509

16 Schmitz AA, Govek EE, Bottner B and Van Aelst L: Rho GTPases: signaling, migration, and invasion. Exp Cell Res 261(1): 1-12, 2000. PMID: 11082269. DOI: 10.1006/excr.2000.5049

17 Kidani T, Yasuda R, Miyawaki J, Oshima Y, Miura H and Masuno $\mathrm{H}$ : Bisphenol A inhibits cell proliferation and reduces the motile potential of murine LM8 osteosarcoma cells. Anticancer Res 37(4): 1711-1722, 2017. PMID: 28373433. DOI: 10.21873/anticanres.11503

18 ACCS_project. Available at: https://github.com/KPU-MASATOY/ACCS_project/ (Last accessed on 12 November 2019)

19 Matsuda H, Nakamura S, Nakashima S, Fukaya M and Yoshikawa M: Biofunctional effects of thiohemiaminal-type dimeric sesquiterpene alkaloids from nuphar plants. Chem Pharm Bull (Tokyo) 67(7): 666-674, 2019. PMID: 31257322. DOI: 10.1248/cpb.c18-01030

20 Yamaguchi $\mathrm{H}$ and Condeelis $\mathrm{J}$ : Regulation of the actin cytoskeleton in cancer cell migration and invasion. Biochim Biophys Acta 1773(5): 642-652, 2007. PMID: 16926057. DOI: 10.1016/j.bbamcr.2006.07.001

21 Ishaq M, Lin BR, Bosche M, Zheng X, Yang J, Huang D, Lempicki RA, Aguilera-Gutierrez A and Natarajan V: LIM kinase 1 dependent cofilin 1 pathway and actin dynamics mediate nuclear retinoid receptor function in T lymphocytes. BMC Mol Biol 12: 41, 2011. PMID: 21923909. DOI: 10.1186/1471-2199-12-41

22 Yang $\mathrm{N}$ and Mizuno K: Nuclear export of LIM-kinase 1, mediated by two leucine-rich nuclear-export signals within the PDZ domain. Biochem J 338( Pt 3): 793-798, 1999. PMID: 10051454.

23 Hotulainen P, Paunola E, Vartiainen MK and Lappalainen P: Actin-depolymerizing factor and cofilin-1 play overlapping roles in promoting rapid $\mathrm{F}$-actin depolymerization in mammalian nonmuscle cells. Mol Biol Cell 16(2): 649-664, 2005. PMID: 15548599. DOI: $10.1091 / \mathrm{mbc}$.e04-07-0555 
24 Song H, Wang Y, Li L, Sui H, Wang P and Wang F: Cucurbitacin E inhibits proliferation and migration of intestinal epithelial cells via activating cofilin. Front Physiol 9: 1090, 2018. PMID: 30131725. DOI: 10.3389/fphys.2018.01090

25 Li D, Wang H, Song H, Xu H, Zhao B, Wu C, Hu J, Wu T, Xie D, Zhao J, Shen Q and Fang L: The microRNAs miR-200b-3p and miR-429-5p target the LIMK1/CFL1 pathway to inhibit growth and motility of breast cancer cells. Oncotarget $8(49)$ : 85276-85289, 2017. PMID: 29156719. DOI: 10.18632/ oncotarget.19205

26 Zhao J, Li D and Fang L: MiR-128-3p suppresses breast cancer cellular progression via targeting LIMK1. Biomed Pharmacother 115: 108947, 2019. PMID: 31078043. DOI: 10.1016/j.biopha. 2019.108947
27 Virtanen SS, Ishizu T, Sandholm JA, Loyttyniemi E, Vaananen HK, Tuomela JM and Harkonen PL: Alendronate-induced disruption of actin cytoskeleton and inhibition of migration/ invasion are associated with cofilin downregulation in PC-3 prostate cancer cells. Oncotarget 9(66): 32593-32608, 2018. PMID: 30220968. DOI: 10.18632/oncotarget.25961

Received November 7, 2019

Revised November 13, 2019

Accepted November 14, 2019 